\title{
Article
}

\section{Processing of written irony in Autism Spectrum Disorder: an eye-movement study}

Au Yeung, Sheena, Kaakinen, J.K., Liversedge, Simon Paul and Benson, Valerie

Available at http://clok.uclan.ac.uk/22354/

Au Yeung, Sheena, Kaakinen, J.K., Liversedge, Simon Paul ORCID: 0000-00028579-8546 and Benson, Valerie ORCID: 0000-0002-0351-4563 (2015)

Processing of written irony in Autism Spectrum Disorder: an eye-movement study. Autism Research, 8 (6). pp. 749-760. ISSN 1939-3792

It is advisable to refer to the publisher's version if you intend to cite from the work. http://dx.doi.org/10.1002/aur.1490

For more information about UCLan's research in this area go to http://www.uclan.ac.uk/researchgroups/ and search for <name of research Group>.

For information about Research generally at UCLan please go to http://www.uclan.ac.uk/research/

All outputs in CLoK are protected by Intellectual Property Rights law, including Copyright law. Copyright, IPR and Moral Rights for the works on this site are retained by the individual authors and/or other copyright owners. Terms and conditions for use of this material are defined in the policies page.

\section{CLoK}

Central Lancashire online Knowledge www.clok.uclan.ac.uk

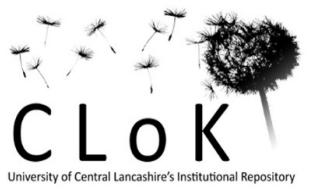


Au-Yeung-Irony Processing in Autism Spectrum Disorders 1

Running head: PROCESSING IRONY IN AUTISM SPECTRUM DISORDERS

Processing of Written Irony in Autism Spectrum Disorder: An Eye-movement Study

Sheena K. Au-Yeung ${ }^{1}$, Johanna K. Kaakinen ${ }^{2}$, Simon P. Liversedge ${ }^{1}$, Valerie Benson ${ }^{1}$

\section{${ }^{1}$ University of Southampton}

${ }^{2}$ University of Turku

Address:

School of Psychology,

Shackleton Building,

University of Southampton,

SO171BJ

Tel: 02380594596

Corresponding Author: Valerie Benson

e-mail: V.Benson@ soton.ac.uk

Grant sponsor: ESRC; Grant number: ES/1019723/1. 
Au-Yeung-Irony Processing in Autism Spectrum Disorders 2

\section{Lay Abstract}

Irony refers to when a statement is made that is opposite to the intended meaning, and is usually used to highlight a failed expectation (e.g. saying "what lovely weather" when it is actually pouring with rain outside). This study investigated whether individuals with Autism Spectrum Disorder (ASD) are able to understand written irony. We recorded the eye movements of typically developing (TD) adults and adults with ASD when they read utterances that could be interpreted ironically or literally, depending on the context of the passage. We found that participants with ASD were as accurate as TD participants at determining whether a speaker meant what they said. Eye movements showed that both participant groups spent longer reading the speaker's statement and the context information that followed it when the statement was ironic compared to when it was meant literally. However, individuals with ASD spend more time overall re-reading text, regardless of whether the statement was ironic or not, suggesting that they do not have a specific difficulty in understanding irony. 


\begin{abstract}
Previous research has suggested that individuals with Autism Spectrum Disorders $\left(\mathrm{ASD}_{2}\right.$ have difficulties understanding others communicative intent and with using contextual information to correctly interpret irony. We recorded the eye movements of typically developing (TD) adults and adults with Autism Spectrum Disorders (ASD adults) when they read speakers'statements that could either be interpreted as ironic or non-ironic depending on the context of the passage. Participants with ASD performed as well as TD controls in their comprehension accuracy for speaker's statements in both ironic and non-ironic conditions. Eye movement data showed that for both participant groups, total reading times were longer for the critical region containing the speaker's statement and a subsequent sentence restating the context in the ironic condition compared to the non-ironic condition. The results suggest that more effortful processing is required in both ASD and TD participants for ironic compared with literal non-ironic statements, and that individuals with ASD were able to use contextual information to infer a non-literal interpretation of ironic text. Individuals with ASD however spent more time overall than TD controls re-reading the passages, to a similar degree across both ironic and non-ironic conditions, suggesting that they either take longer to construct a coherent discourse representation of the text, or that they take longer to make the decision that their representation of the text is reasonable based on their knowledge of the world.
\end{abstract}

Key words: Irony, figurative language, Autism, eye movements, weak central coherence, complex information processing 
Au-Yeung-Irony Processing in Autism Spectrum Disorders 4

\section{Introduction}

Autism Spectrum Disorder (ASD) is a neurodevelopmental condition defined by qualitative impairments in social communication and restricted, repetitive, and stereotypical patterns of behaviour and interests (APA, DSM-V, 2013). Although it is usually reported that high-functioning individuals with ASD develop an adequate level of simple procedural language skills, abnormalities in complex interpretative and inferential abilities in the language domain have also been documented (e.g. Minshew, Goldstein, \& Siegel, 1995). The purpose of the current study was to explore how individuals with ASD process written language, where the interpretation of what is being read could have ambiguous meaning, namely written irony.

Ironic statements embedded in a text passage, such as "I see you are very smart" presented in a context in which a friend achieved the lowest grade in his class, typically causes readers some disruption in reading. Typically Developed (TD) readers may initially interpret the utterance as literal, but when they realize that a non-ironic meaning does not fit with the context in which the utterance was presented, an ironic reinterpretation has to be made (Giora, 1995; Searle, 1993, as cited in Evans, 2010). For example, Filik and Moxey (2010) presented participants with short passages of text containing an utterance, which could either be interpreted as ironic or non-ironic depending on the preceding context of the sentence, and recorded readers' eye movements to examine online processing of the ironic vs. non-ironic utterances. Participants spent longer reading the same statement when it was meant ironically compared to when it was meant literally. Filik, Leuthold, Wallington, and Page (2014) further investigated how individuals process familiar and unfamiliar written irony, and found that readers showed longer first-pass reading times and total reading times on the disambiguating word, ("liked" in the example above) and on the remainder of the sentence that followed the disambiguating word ("your story") for unfamiliar ironic 
sentences compared to non-ironic control sentences. Longer regression path times for the end of the sentences were also found for the unfamiliar ironic sentences, indicating that readers reread the ironic passages after encountering an ironic utterance in that passage. Recently, Kaakinen, Olkoniemi, Kinnari, \& Hyönä, (2014) found that irony triggered a higher probability, and longer duration, of immediate re-reading of the ironic target utterance, and shorter look-backs to the context region compared to when the target utterance was presented

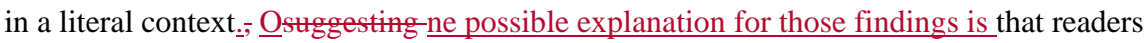
selectively re-read the ironic part of the text in order to re-interpret and confirm the ironic interpretation of the text.

Comprehending written irony requires that the reader understands that the literal meaning does not make sense in the context in which it is presented. This means that the reader has to be able to maintain and make use of the contextual information. Second, the reader has to empathise with the mental state of the speaker in order to make the inference that the utterance was intended as ironic. Finally, the reader has to be able to integrate the initially ambiguous utterance into the developing memory representation of the passage. Considering that ASD has been linked with problems in using contextual information (Happé, 1999), deficits in Theory-of-Mind (Baron-Cohen, 2001), as well as problems with complex information processing in general (Minshew \& Goldstein, 1998), comprehending irony could be regarded as potentially challenging for individuals with ASD.

However, findings from previous studies on irony processing with ASD participants are inconsistent. It has been found that children and adolescents with high functioning autism or Pervasive Developmental Disorders Not Otherwise Specified were impaired compared to TD controls at interpreting ironies and metaphors, and that performance was related to their theory-of-mind and verbal abilities (de Villiers et al., 2011). This was not the case for individuals with Asperger's Syndrome, who performed as well as TD controls on theory-of- 
mind tasks, metaphor understanding, irony judgments and explanations. These abilities were suggested to be attributable to the high verbal IQ of participants with Asperger's Syndrome (Diaz, 2010).

It has also been shown (Gyori, 2006) that individuals with ASD across a wide age range (9 to 43 years), and verbal IQ range (63-117), were less accurate and took longer than TD controls in responding to comprehension questions regarding whether stories were ironic or not, although performance of the participants with ASD was still surprisingly higher than expected. Other reports support the idea that the nature of processing is different in ASD compared to TD individuals. For example, Pexman et al. (2011) found that high-functioning children with ASD performed as accurately as TD controls in their ability to point to an object associated with a speaker's ironic intent in an irony comprehension task that minimized verbal and pragmatic demand. However, differences in judgement latencies, eye gaze and humour evaluations suggested that irony was processed in a different way in the ASD relative to the TD group.

Wang, Lee, Sigman, and Dapretto (2006) found that children and adolescents with Autism and Asperger's Syndrome (IQ > 70) were less accurate than TD controls in judging whether statements in spoken scenarios were sincere or ironic in text types where knowledge of event outcome was available, although both ASD and TD participants performed significantly above chance. In contrast, a later study by the same group of authors (Wang, Lee, Sigman, \& Dapretto, 2007) reported no group differences in accuracy and response time. Using the same stimuli and participants matched in all IQ measures (IQ > 75), Colich, Wang, Rudie, Hernandez, Bookheimer, and Dapretto (2012) again found equivalent accuracy performance for both groups across ironic and sincere conditions. These studies however, have been criticised on a number of grounds, not least because of their often contradictory interpretation of findings in relation to brain activity during task performance. 


\section{The present study}

The aim of the current study was to gain some insight into how individuals with ASD process irony, and to compare this with what we know about neurotypical processing of irony. In a partial replication of Filik and Moxey's (2010) study participants read short passages containing an utterance that could either be interpreted ironically or non-ironically, depending on the preceding context and comprehension questions tested the interpretation of the utterances. Consistent with Filik and Moxey's findings (2010), we predicted that TD participants would show longer re-reading times for the critical utterance for the ironic compared to the non-ironic condition. We also predicted that TD participants should spend more time re-reading the contextual information for the ironic condition compared to the nonironic condition, in order to resolve the incongruent information between the context and the literal meaning of the utterance.

In relation to ASD, Weak Central Coherence Theory (Happé, 1999) predicts that ASD participants would not take into account contextual information whilst reading the sentences due to impaired global processing. The prediction therefore would be that the ASD participants should take the ironic utterance literally and ignore the factual inconsistency between the context and the ironic utterance. Impaired ability to comprehend the ironic statement should be reflected in the offline accuracy data in the comprehension task for the ironic utterances. For the eye movement data we would expect no difference in the way participants with ASD process ironic and non-ironic versions of the text, and this should be reflected by equivalent reading times between the ironic and non-ironic condition in the critical utterance and the contextual information in the text passages.

The Disordered Complex Information Processing Theory would predict that participants with ASD should have a generalised deficit in complex tasks with "high 
requirements for on-line information processing" (Minshew et al 1992; Minshew et al., 1994, as cited in Minshew et al., 1995). Based on previous findings (Benson et al., 2012, AuYeung et al., 2013), we expected that our high-functioning sample of participants with ASD would not necessarily show impaired performance in their offline comprehension question responses specifically for the ironic condition, rather they would show intact performance for both ironic and non-ironic conditions, comparable to those of TD participants. However, if complexity could be characterised in terms of the figurative nature of the language, then online eye movement measures should reveal difficulties in interpreting ironic utterances over and above those experienced by TD individuals. Specifically, if there is a difference in the time-course in which irony is resolved between the groups then ASD individuals might be expected to take disproportionately longer to resolve the irony than TD individuals. We would therefore expect to find that the irony effect (significant difference between the ironic and non-ironic text type) to be greater for the ASD group in the total time for the critical region and the contextual information.

\section{Method}

\section{Participants}

In total forty two volunteers participated in the study; 20 in the TD group and 22 in the ASD group. Participants were clinically diagnosed prior to the study and recruited from the National Autistic Society website, Children on the Autism Spectrum Parents' Association, and a database of volunteers who had previously taken part in other studies at the University of Southampton. Sixeme participants had to be excluded from the analyses, for the following reasons. One participant (male) in the ASD group was excluded because he was unable to provide formal evidence of a clinical diagnosis. One other participant (male) in the ASD group did not complete the IQ assessment. One TD participant (male) and two ASD 
participants ( 2 females) were excluded due to scoring below 90 on at least one measure in IQ subscales. Finally, one participant (female) with ASD was excluded due to eye tracker calibration error. The demographics of the final sample are presented in Table 1, which included 19 participants in the TD group (13 males, 6 females) and 18 participants in the ASD group (16 males, 2 females). As a result of having to exclude some of the participants from the analysis of our data, the ASD group had a slightly greater age mean than the TD group, but the majority of participants fall within a similar age range (TD $m d n: 21, m d n$ : ASD: 28). The two participant groups were group matched on all measures of the Wechsler Abbreviated Scale of Intelligence (The Psychological Corporation, 1999). However, as expected, the ASD group scored significantly higher on Autism-Spectrum Quotient (AQ: Baron-Cohen, Wheelwright, Skinner, Martin, \& Clubley, 2001) compared to the TD group.

\section{[INSERT TABLE 1 HERE]}

\section{Materials}

The experimental stimuli consisted of 36 short text passages, each made up of three sentences. There were two versions of each passage, an ironic version and a non-ironic version. Some of the passages were modified stimuli from Filik and Moxey’s study (2010) and some passages were written by one of the authors. An example story is presented below:

John and Mary were sitting in the newspaper office, reading through a huge pile of $f_{1}$ hate mail / fan mail.2 | 'Obviously our readers ${ }_{3}$ | liked your story',4 $\mid$ said John.5 | Mary was surprised that ${ }_{6} \mid$ so few / many people liked her news article.7

Each passage was divided into regions of interest that were used for eye movement analyses. The first sentence of the passage contains the context region (2), which is the end portion of the sentence and it differs for the ironic and non-ironic version of the story ("hate 
mail" vs. "fan mail"). The critical region (4) in the second sentence contains the ironic or non-ironic utterance as determined by the previous context, and the short phrase immediately following the critical region was labelled as the spill-over region (5). The last sentence of the story contained a context restatement region (7), in which the contextual information was restated (so few vs. so many people liked her news article) for both the ironic and non-ironic passages. To be clear the final sentence wais always a restatement of the first context setting sentence. Since these sections of the sentence should drive the reading time effects that we predicted, we only report analyses for these regions.

The text was presented with size 14 Courier New black font on a white background. Triple spacing between each line of text was used to ensure clear distinction between fixations on different lines.

The experimental stimuli were divided into two lists (A \& B). Each list contained 18 ironic passages and 18 non-ironic passages that were intermixed. If the ironic version of a passage was in List A, then the non-ironic counterpart would be in List B. An additional 32 filler passages were added to each list. These were identical for the two lists and were mixed in with the experimental stimuli and presented in random order. Participants were randomly assigned to view stimuli from either list A or B. There were four practice trials before the experimental stimuli were presented. In all, each participant viewed a total of 72 passages (Stimuli are available from the corresponding author on request).

Comprehension questions were presented for a third of the stimuli. The inclusion of Formatted: Indent: Left: $0 \mathrm{~cm}$, Line spacing: Double these was designed to gauge participants' understanding of the intended meaning of the text. The comprehension questions assessed the literal meaning for the non-ironic passages and the ironic meaning for the ironic passages. The questions asked participants whether the speaker meant what they said in the statement region of the sentence: For example, if the statement was "Clearly the readers liked your story" within the context of a writer receiving hate mail 
Au-Yeung-Irony Processing in Autism Spectrum Disorders 11

from the readers, and a colleague (in this case John) made an ironic comment, then the question would be " Did John think the readers liked the story?"

Participants viewed the stimuli binocularly from a screen using 1024 x 768 resolution. Eye movements were recorded monocularly for the right eye using an Eyelink 1000 eye tracker (SR Research Ltd, Osgoode) with a viewing distance of $70 \mathrm{~cm}$. Participants placed their head on a chin rest and forehead support to stabilise their position during the experiment. Calibration was done using a nine-point matrix. A fixation dot was presented at the beginning of each trial indented to the left of the first word of each passage; participants were required to fixate this dot before the text appeared on the screen. Once a participant's fixation matched the position of the dot, the experimenter pressed a key to release the stimuli onto the screen. Participants were recalibrated if the fixation drifted away from the fixation dot between trials.

\section{Design and Procedure}

The experiment was a mixed design with a within-participant variable Text Type (Ironic vs Non-ironic) and a between-participant variable Group (TD vs ASD).

Procedure

\section{Results}

\section{Comprehension Response Accuracy}

The comprehension scores (counts) are presented in Table 2. A 2 Text Type (Ironic vs Non-Ironic) x 2 Group (TD vs ASD) ANOVA was computed on accuracy score to comprehension questions. There was a significant main effect of Text Type, $F(1,35)=$ 
$11.126, p=.002, \eta_{\mathrm{p}}^{2}=.241$, indicating that participants were significantly more accurate when answering comprehension questions after non-ironic $(M=5.376, S E=.153)$ compared to ironic texts $(M=4.365, S E=.295)$. There was no significant main effect of Group, $F(1,35)$ $=2.564, p=.118, \eta_{\mathrm{p}}^{2}=.068$, nor was there a significant interaction between Text Type and Group, $F(1,35)=1.563, p=.220, \eta_{\mathrm{p}}{ }^{2}=.043$, suggesting that both groups found it more difficult to interpret ironic than non-ironic utterances. PImpertantly, participants with ASD performed similarly, statistically, to TD controls in the ironic as well as non-ironic condition ${ }_{2}-$ however, it should be noted that there was a greater numerical difference between ASD and Formatted: Font: (Default) Times New Roman, 12 pt, Not TD for the ironic, compared to the non-ironic condition with respect to accuracy.

\section{[INSERT TABLE 2 HERE]}

\section{Eye Movements during Passage Reading}

Data trimming. For the eye movement data, any fixations that were shorter than 50 ms were either removed, or were merged with a nearby fixation if that fixation was within one degree of the target fixation. In all data analyses, observations deviating more than three standard deviations from the condition mean, computed separately for each participant, were excluded (see Appendix).

Eye movement measures. Three different eye movement measures were computed for the regions of interest presented above. First pass reading time is the summed duration of the fixations in a region of interest until the reader moved his/her eyes to fixate another region. Regression path reading time is the summed duration of the fixations that occurred from the first fixation in a region until the participant moved their eyes beyond that region to the right. Therefore, regression path reading time included all the fixations in a region and any regressive fixations on words in the previous portion of the text until a fixation to the right of the region. Total reading time is the summed duration of all the fixations in a region. 
First-pass reading times gives an indication of early stages of linguistic processing; regression path reading time reflects early processing difficulty as well as time spent re-inspecting the text in an effort to recover from any initial difficulty; and total reading time provides a measure of overall processing difficulty associated with a portion of the sentence (Filik \& Moxey, 2010).

Data analysis. The eye movement data were analysed with linear mixed effects models (Baayen, Davidson, \& Bates, 2008) using the lme4 package (Bates, Maechler, Bolker, \& Walker, 2013) available for R statistical software (R Core Team, 2013). Participant group (ASD vs. TD) and Text type (ironic vs. non-ironic) were deviation coded and entered into the models together with their interaction term as fixed effects. Participants and items were entered into the models as crossed random effects; the random slope for text type was entered at the participant level, and the random slopes for text type, participant group and their interaction was entered into the models at the item level (Barr, Levy, Scheepers, \& Tily, 2013). P-values for the fixed effects estimates were computed using the lmerTest package (Kuznetsova, Bruun Brockhoff \& Haubo Bojesen Christensen, 2013). T-values > 1.96 were taken as indicators of signifieant effects at $\alpha=.05$ level. Detailed information of the fitted models is presented in Appendix 4 .

Context region. The context setting phrase determines whether or not the text that follows should be read as ironic or non-ironic. The descriptive statistics for this region are presented in Table 3. We expected that no effects of irony would be apparent for first-pass reading of the context region, nor in the regression path times, as at this point the ironic utterance had not yet been encountered. However, if readers try to resolve the ironic meaning of the subsequent text by re-reading the text in the context region, total reading times should be longer for this region of ironic in comparison to non-ironic texts. 
There were no statistically significant effects in the first-pass fixation time, regression path duration or total fixation time on the context region, all $|t|$ 's $<1.84$. However, as can be seen from the means, the ASD group showed a tendency for longer total fixation times for the context region of the sentence $(\underline{B=439, t=1.84, p=.0692)})$, indicating that the readers in the ASD group spent longer re-reading the context setting sentence (for both ironic and nonironic sentences) than the TD group.

Critical region. The descriptive statistics for the eye movement measures in the critical region are presented in Table 4. There were no significant effects in the models for the first-pass reading time or the regression path duration $(t$ 's $<1.5)$. The analysis of the total reading time showed that ironic phrases received longer total fixation times than non-ironic

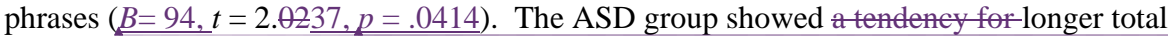
readingtimesthan the TDgroup buttheeffectlidnetrechsignificance $(B=290, t=1.80228, p=.0292)$. Therewasalsenosignificant interaction between Text Type and Group $(t=.084)$.

\section{[INSERT TABLE 4 HERE]}

Spill-over region. The spill-over region is the phrase immediately following the critical region and fixations in this region might reflect slightly delayed processing associated with the computation of irony. The descriptive statistics for the eye movement measures for the spill-over region are presented in Table 4.

There were no significant effects in the models for the first-pass reading time or for the regression path duration on the spill-over region $(|t|$ 's $<1.5)$. The analysis of the total reading time showed that the ASD group had longer total reading times than the TD group in this region $(B=247, t=2.77, p=.00818)$. 
Restatement region. In the restatement region, the contextual information is restated. Detection and attempts to make sense of the incongruent information between the context and the ironic utterance might be reflected in elevated reading times in this region. The descriptive statistics are presented in Table 5.

There were no effects in the first-pass reading time on the restatement region $(t$ 's $<1)$. However, a number of mMain eEffects were observed for other measures. Regression path times were longer in the ironic than non-ironic condition $(B=268, t=2.31, p=.02798)$, and Importantly, however, the ASD group showed longer regression path times at this region than the TD group $(\underline{B=1248, t=3.71509, p=.00387)})$. Total fixation times were longer in the ironic than in the non-ironic condition $(t=2.40)$ and the iIndividuals with ASD also showed longer total fixation durations in the restatement region than the TD individuals $(\underline{B=369, t}=$ $2.50, p=.0287)$

Formatted: Font: Italic
Formatted: Font: Italic
Formatted: Font: Italic
Formatted: Font: Italic
Formatted: Font: Italic

\section{[INSERT TABLE 5 HERE]}

\section{Discussion}

The current study examined the time-course of irony processing in both TD individuals and individuals with ASD. Consistent with previous eye movement studies (Filik \& Moxey, 2010; Kaakinen et al., 2013), for the ironic sentences our TD participants produced longer total reading times for the critical sentences containing the utterance, and also longer total reading times for sentences that restated the contextual information that had preceded the utterance-..When the utterance was intended to be ironic compared to when it was to be taken literally. These findings support the view that extra processing is involved when reading written irony, and there were evidence suggesting that this is due to the need to reject the more salient literal surface meaning of an utterance and infer its non-literal ironic 
meaning (Filik \& Moxey, 2010). The findings for the ASD group are discussed below in relation to two current contemporary theories of ASD.

\section{Weak Central Coherence}

Weak Central Coherence Theory predicts that individuals with ASD would ignore the context information in the passages and as such, would fail to notice the irony and hence maintain the literal non-ironic interpretation of the utterance. Our findings did not support this view. Firstly, impaired ability to correctly interpret the intended meaning of the ironic utterance should lead to a disproportionate decline in accuracy in the comprehension questions that followed an ironic text. Our results showed that although both participant groups were less accurate in responding to the comprehension questions after reading ironic than non-ironic texts, participants with ASD did not show a disproportionately poorer performance than the TD participants for the ironic condition. Secondly, if participants with ASD were interpreting both ironic and non-ironic versions of the utterance literally, then there should be no differences between the two text types in reading times for the critical regions. In fact, our results showed that participants with ASD, similar to TD participants, spent more time in total reading the text in the critical region for the ironic compared to the non-ironic condition. Thirdly, any inability to appreciate the inconsistency between the ironic utterance and the contextual information should have been reflected in equivalent reading times for the context region and the context restatement region between ironic and non-ironic conditions within the ASD group. However, neither participant group spent more time in total reading the contextual region for the ironic compared to the non-ironic condition. We also found that both participant groups spent more time re-reading previous portions of the text, and spent more time in total reading the text, in the context restatement region in the ironic compared to the non-ironic condition. This in itself means that ASD and TD 
participants noticed the inconsistency between the ironic utterance and the context when it was restated, and consequently went back to re-read the text associated with the inconsistency.

\section{Disordered Complex Information Processing}

We also tested the assumption that if complexity increases with figurativeness then individuals with ASD should show elevated difficulty with processing ironic language, compared to TD individuals. In the current study, the non-ironic condition can be considered the simpler task and the ironic condition can be considered the more complex task as it requires more complex processing during which participants must negate the literal meaning of the utterance to compute the intended ironic interpretation. We predicted that the ASD group would require substantially more effort to process and integrate incongruent information in the discourse compared to the TD group which would lead to elevated rereading in ASD relative to TD participants for the contextual information, the critical utterance, and the restatement of the contextual information for the ironic condition relative to the non-ironic condition. We found however that both groups showed typical disruption when processing ironic text. Furthermore the re-reading findings from the statement region and the context restatement region suggest that ironic meaning is not automatically accessed for either participant group, but that some delayed processing associated with reinterpretation of the literal text meaning has to be carried out. Thus the findings appear to support neither Weak Central Coherence claim that individual with ASD are impaired at using contextual information to disambiguate meaning, and nor do they support the Disordered Complex Information Theory proposal that ASD have more difficulty associated with complex processing tasks, when complexity is operationally defined as the figurativeness of language, as in the current study. 
One unexpected finding in the current study was that there were elevated total reading times across several regions for ASD readers compared with TD readers regardless as to whether the text was ironic or non-ironic. In a sentence-by-sentence self-paced reading task (Gyori, 2006), participants read several context sentences followed by a target utterance that was either ironic or literal. During this task, participants were required to press a button after reading each sentence to trigger the presentation of the next sentence. They were then required to respond to a forced choice question about whether an interpretation of the target utterance was true or not at the end of each trial. It was found that both ASD and TD participants consistently showed prolonged reaction times to the interpretation question for ironic compared to a literal condition. However, and consistent with the current study, participants with ASD had greater reading times for all context sentences and the target utterance, as well as greater reaction time to the interpretation question, across literal and ironic conditions compared to TD participants.

Considering the similarities in the time-course of processing for text materials containing irony between our ASD and TD participants, it is unsurprising that there was also no between-group difference in comprehension accuracy for ironic materials in the current study. Some previous irony studies (e.g. Gyori, 2006; Wang et al., 2006) had however reported lower accuracy for comprehension, and hence have concluded that individuals with ASD have difficulties in interpreting communicative intents of others. One drawback of these studies however was that their comprehension accuracy measures were collapsed across ironic and non-ironic conditions; therefore it is unclear as to whether this comprehension deficit is specifically related to the complexity of the ironic text materials. In a more recent study by Colich et al. (2012), in which comprehension rates were calculated separately for ironic and sincere conditions, equivalent accuracy was found between ASD and TD groups for both ironic and sincere conditions. This is consistent with the current study, potentially 
suggesting that there could be a lack of processing difficulty associated with irony specifically.

Our results overall indicate that it is not the case that individuals with ASD were unable to integrate information and make correct interpretation of the sentences on-line as they read them. If that had been the case we would have observed a delay in detection of the irony in the ASD group, in the eye movement measures compared to the TD group. However, an identical time-course of irony detection between ASD and TD participants confirmed that this was not the case. Instead, there was an overall increase in reading times regardless of whether irony was or was not present in the ASD group. One explanation for the current findings is that individuals with ASD were taking extra time to be sufficiently assured that their interpretation of the sentence was correct. Only when this was complete did the participants with ASD feel able to terminate the display to move on to either a comprehension question or the next passage. This account is less related to language comprehension processes, but more about the time it takes participants with ASD to develop confidence in their interpretation of the text.

In conclusion, our results showed that individuals with ASD are just as able as TD individuals to use contextual information to aid comprehension of irony, and there was no difference in the time-course of irony detection, as reflected by eye movement measures, to indicate processing difficulty specifically related to figurativeness of irony in ASD. This is consistent with various studies by Norbury $(2004,2005 a, 2005 b)$ who has shown that specific language impairment rather than autistic status is a factor that contributes to the ability to understand non-literal language such as metaphors and idioms and ambiguous terms. The main difference between our TD and ASD group was that there were longer re-reading times for all regions of interest in the text, and for both conditions, in the ASD group. Although it remains to be empirically tested, we think that the re-reading in the ASD group could reflect 
Au-Yeung-Irony Processing in Autism Spectrum Disorders 20

an increased need to recheck the text, to be confident that the interpretation of the utterance was correct, given that comprehension questions followed some of the passages. 
Au-Yeung-Irony Processing in Autism Spectrum Disorders 21

\section{Acknowledgements}

This work was supported by the Economic and Social Research Council (ESRC Reference Number: ES/1019723/1). We would like to thank Dr Ruth Filik for providing the original version of the text stimuli used in her study (Filik \& Moxey, 2010). The authors declare no conflict of interest. 
Au-Yeung-Irony Processing in Autism Spectrum Disorders 22

\section{References}

American Psychiatric Association (2013). Diagnostic and statistical manual of mental disorders (5th ed.). Arlington, VA: American Psychiatric Publishing.

Au-Yeung, S. K., Benson, V., Castelhano, M., \& Rayner, K. (2011). Eye movement sequences during simple versus complex information processing of scenes in autism spectrum disorder. Autism Research and Treatment. doi:10.1155/2011/657383.

Au-Yeung, S. K., Kaakinen, J. K., \& Benson (2013). Cognitive perspective-taking during scene perception in Autism Spectrum Disorder: Evidence from Eye Movements. Autism Research, 7, 84-93. doi: 10.1002/aur.1352

Baron-Cohen, S. (2001). Theory of mind in normal development and autism. Prisme, 34, 174-183.

Baron-Cohen, S., Wheelwright, S., Skinner, R., Martin, J., \& Clubley, E. (2001). The autism spectrum quotient (AQ): evidence from Asperger Syndrome/High Functioning Autism, males and females, scientists and mathematicians. Journal of Autism and Developmental Disorders, 31, 5-17.

Baron-Cohen, S., Wheelwright, S., Hill, J., Raste, Y., \& Plumb, I. (2001). The "reading the mind in the eyes" revised version: a study with normal adults, and adults with Asperger Syndrome or High-functioning Autism. Journal of Child Psychology and Psychiatry, 42, 241-251. doi: 10.1111/1469-7610.00715

Benson, V., Castelhano, M., Au-Yeung, S. K., \& Rayner, K. (2012). Eye movements reveal no immediate "WOW" ("which one's weird") effect in Autism Spectrum Disorder. The Quarterly Journal of Experimental Psychology. doi: $10.1080 / 17470218.2011 .644305$ 
Colich, N. L., Wang, A. T., Rudie, J. D., Hernandez, L. M., Bookheimer, S. Y. \& Dapretto, M. (2012). Atypical neural processing of ironic and sincere remarks in children and adolescents with autism spectrum disorders. Metaphor and Symbol, 27(1), 70 -92. doi: $10.1080 / 10926488.2012 .638856$

de Villiers, P. A., de Villiers, J. G., Diaz, S., Cheung, C., Alig, R., \& Raditz, V. (2011, November) Non-literal language and theory of mind in Autism Spectrum Disorders. Poster presented at the American Speech-Language-Hearing Association Convention, San Diego, CA. Retrieved from http://www.asha.org/Events/convention/handouts/2011/de-Villiers-de-Villiers-DiazCheung-Alig-Raditz-Paul/

Diaz, S. (2010). Understanding metaphors, irony and sarcasm in high functioning children with autism spectrum disorders: its relationship to theory of mind (thesis). Retrieved from https://dspace.smith.edu/handle/11020/15128?show=full

Evans, V. (2010). Figurative language understanding in LCCM theory. Cognitive Linguistics, 21(4), 601-662. doi: 10.1515/COGL.2010.020

Filik, R., Leuthold, H., Wallington, K., \& Page, J. (2014). Testing theories of Irony Porcessing using eye-tracking and ERPs. Journal of Experimental Psychology: Learning, Memory, and Cognition, 40(3), 811-828. doi: 10.1037/a0035658

Filik, R. \& Moxey, L. M. (2010). The on-line processing of written irony. Cognition, 116, 421-436. doi:10.1016/j.cognition.2010.06.005

Gernsbacher, M. A. \& Pripas-Kapit, S. R. (2012) Who's missing the point? a commentary on claims that autistic persons have a specific deficit in figurative language 
Au-Yeung-Irony Processing in Autism Spectrum Disorders 24 comprehension. Metaphor and Symbol, 27(1), 93-105, doi:

$10.1080 / 10926488.2012 .656255$

Gibbs, R. W. (1986). On the psycholinguistics of sarcasm. Journal of Experimental Psychology: General, 118, 3-15.

Giora, R. (1995). On irony and negation. Discourse Processes, 19, 239-264.

Gyori, M., Lukacs A., Pléh, C. (2004): Towards the understanding of the neurogenesis of social cognition: evidence from impaired populations. Journal of Cultural and Evolutionary Psychology, 2, 261-282. doi:10.1556/JCEP.2.2004.3-4.6

Gyori, M. (2006). Autism and Cognitive Architecture. Budapest, Hungary: Akadémiai Kiadó.

Happé, F. G. E. (1999). Autism: cognitive deficit or cognitive style? Trends in Cognitive Sciences, 3(6), 216-222. doi: 10.1016/S1364-6613(99)01318-2

Kaakinen, J. K.,Olkoniemi, H., Kinnari, T., \& Hyönä, J. (2014). Processing of Written Irony: An Eye Movement Study. Discourse Processes, 51(4), 287-311. doi:

$10.1080 / 0163853 X .2013 .870024$

Minshew, N. J., \& Goldstein, G. (1998). Autism as a disorder of complex information processing. Mental Retardation and Developmental Disabilities Research Reviews, 4(2), 129-136. doi: 10.1002/(SICI)1098-2779(1998)4:2<129::AID-

MRDD10>3.0.CO;2-X

Minshew, N.J., Goldstein, G., Siegel, D.J. (1995). Speech and language in high functioning autistic Individuals. Neuropsychology. 9(2), 255-261. 
Minshew, N. J., Williams, D. L., \& McFadden, K. (2008). Information processing, neural connectivity, and neuronal organization. In A. W. Zimmerman (Ed.), Autism: Current Clinical Neurology (pp. 381-405). Totowa, NJ: Humana Press.

Norbury, C. F. (2004). Factors supporting idiom comprehension in children with communication disorders. Journal of Speech, Language, and Hearing Research, 47, 1179-1193. doi:10.1044/1092-4388(2004/087)

Norbury, C. F. (2005a). Barking up the wrong tree? Lexical ambiguity resolution in children with language impairments and autistic spectrum disorders. Journal of Experimental Child Psychology, 90, 142-171. doi:10.1016/j.jecp.2004.11.003

Norbury, C. F. (2005b). The relationship between theory of mind and metaphor: Evidence from children with language impairment and autistic spectrum disorder. British Journal of Developmental Psychology, 23, 383-399. doi:10.1348/026151005X26732

Ozonoff, S., \& Miller, J. N. (1996). An exploration of right-hemisphere contributions to pragmatic impairments of autism. Brain and Language, 52(3), 411-434.

Persicke, A, Tarbox, J, Ranick, J, \& St. Clair, M. (2012). Establishing metaphorical reasoning in children with autism. Research in Autism Spectrum Disorders, 6, 913920. doi:10.1016/j.rasd.2011.12.007

Pexman, P. M., Rostad. K. R., McMorris, C. A., Climie, E. A., Stowkowy, J., \& Glenwright, M. R. (2011). Processing of ironic language in children with high-functioning Autism Spectrum Disorder. Journal of Autism and developmental disorders, 41(8), 1097-1112. doi: 10.1007/s10803-010-1131-7 
Au-Yeung-Irony Processing in Autism Spectrum Disorders 26

The Psychological Corporation (1999). Wechsler Abbreviated Scale of Intelligence (WASI) Manual. San Antonio, TX: The Psychological Corporation.

Wang, A. T., Lee, S. S., Sigman, M., \& Dapretto, M. (2006). Neural basis of irony comprehension in children with autism: The role of prosody and context. Brain, 129, 932-943. doi: 10.1093/brain/aw1032

Wang. A. T., Lee, S. S., Sigman, M., \& Dapretto, M. (2007). Reading affect in the face and voice: Neural correlates of interpreting communicative intent in children and adolescents with autism spectrum disorders. Archives of General Psychiatry, 64, 698708. doi:10.1001/archpsyc.64.6.698 